\title{
Simulations of multipactor breakdown between two cylinders
}

\author{
J. Rasch ${ }^{1}$, V. E. Semenov ${ }^{2}$, E. Rakova ${ }^{2}$, D. Anderson ${ }^{1}$, \\ J. F. Johansson ${ }^{3}$, M. Lisak ${ }^{1}$ and J. Puech ${ }^{4}$ \\ ${ }^{1}$ Chalmers University of Technology, Göteborg, Sweden \\ ${ }^{2}$ Institute of Applied Physics, Nizhny Novgorod, Russia \\ ${ }^{3}$ RUAG Space AB, Göteborg, Sweden \\ ${ }^{4}$ Centre National d'Études Spatiales, 31401 Toulouse Cedex 9, France
}

PACS number 52.80.Pi

\begin{abstract}
Simulations have been performed to determine the multipactor breakdown threshold in a microwave structure comprised of two parallel cylinders, chosen to be an approximate model of an open helix microwave antenna system. The electromagnetic field between the cylinders is available in closed analytical form and a Monte Carlo software has been developed to calculate the 2D electron trajectories and to simulate the multipactor avalanche in this inhomogeneous electric field for different ratios of cylinder radius and distance of separation between the cylinders. The results are compared with those of a recently published analytical theory and show a qualitatively good agreement. In particular, it is confirmed that for given distance between cylinders, there exists a smallest cylinder radius below which no two-sided multipactor breakdown can occur. The basic physical explanation is a loss mechanism for secondary emitted electrons that is caused by the curvature of the cylinder surfaces together with the strong electric field at the surfaces. The results imply that the breakdown threshold in realistic open helix antennas is significantly higher than those predicted using extrapolations based on resonance theory and the classical two parallel plate model.
\end{abstract}




\section{INTRODUCTION}

A significant effort has been made during the last ten years to make possible reliable predictions for multipactor breakdown thresholds in microwave devices with geometries more complex than the classical one involving two parallel infinite plates ${ }^{1-15}$. The analyzed situations typically involve three new complications as compared to the plane parallel plate situation: finite extension in the parallel direction, inhomogeneous electric fields, and curved field lines. This leads to new physical effects that affect the electron motion and the corresponding breakdown condition and which makes extrapolations of the multipactor threshold based on the two parallel plate model unreliable. Examples of such more complicated geometries that have been analyzed are e.g. irises $^{7}, \operatorname{rectangular}^{3,5}$, coaxial $^{1,4,12,13}$, circular $^{1,11}$, and wedge-shaped wave guides ${ }^{14,15}$.

Very little attention has so far been given to multipactor breakdown in open antenna structures, an important example in space applications being helix antennas, which consist of helically wound cylindrical metallic wires. In technically relevant cases, the pitch angle of the helix is small and the geometry can locally be approximated as that of parallel wires. When the distance between the wires becomes large compared to the wire radii, the corresponding electric field becomes strongly inhomogeneous and significant deviations from the plane parallel plate predictions can be expected. A first qualitative analysis of this problem was recently made ${ }^{16}$ where the electron motion in the electromagnetic field between two wires was analyzed by separating the motion into a slowly varying drift velocity (driven by the ponderomotive force due to the electric field inhomogeneity) and a rapidly oscillating part (driven by the oscillating electric field). The curvature of the cylindrical surfaces of emission was shown to give rise to a spreading out of the emitted secondary electrons, which implied a dilution of the electron density equivalent to a loss of electrons. This resulted in a higher multipactor breakdown threshold for the two wire structure than for the classical situation corresponding to the case of two plane parallel infinite plates. The importance of this effect was found to be determined by the ratio of the cylinder radii and the distance between the cylinders and it was shown that when this ratio is small, electron losses become large, and multipactor can only occur for surfaces having very large secondary emission coefficients.

The analysis in Ref.[16] was based on a strong simplification of the electron dynamics 
by considering only motion close to the symmetry axis between the cylinders (where the strongest electric field occurs) and by dividing the electron motion into two components with well separated time scales. The main result was a prediction for the lower threshold for multipactor breakdown that turned out to be significantly higher than that given by extrapolations based on the plane parallel plate theory. In particular, it was found that under a certain cylinder radius, which depended on the distance between the cylinders and the maximum value of the secondary emission yield, double sided multipactor becomes impossible, due to the strong losses caused by the geometrical dispersion of electrons during successive passages between the cylinder surfaces. The accuracy of these predictions was difficult to assess and therefore it is important to carry out a comparison with full numerical simulations. This is the purpose of the present analysis where an extensive numerical effort has been done in order to simulate the multipactor avalanche in the two wire system taking into account the exact analytical form of the electromagnetic field. The aim is to understand the influence of the wire dimensions on the multipactor threshold and to compare the corresponding results with those of the previous approximate theory. A special software was developed to study the electron trajectories and to simulate the multipactor avalanche in a two wire system where the fundamental TEM mode is excited. The software takes into account a spread of electron emission velocity and is based on a Monte-Carlo algorithm and Vaughan's approximation ${ }^{17}$ for the secondary emission yield. The results are compared with those of the previous theory and also with those of classical resonance theory. Good qualitative agreement between theory and simulations is found. In particular, the prediction of a lower radius under which double sided multipactor becomes impossible is confirmed. However, neither the non-resonant theory nor the resonant theory agree completely with the simulation results and the physical reasons for this discrepancy are identified and discussed.

\section{FIELD CONFIGURATION}

As previously mentioned, for low pitch angles, a helix antenna can be approximated as consisting of two parallel infinite metal cylinders. In this geometry, multipactor analysis is simplified by the fact that the electric and magnetic fields can be determined analytically using logarithmic potential theory ${ }^{18}$. The electromagnetic field travels along the two wire system in the form of a purely transverse electromagnetic (TEM) wave containing both 
electric and magnetic components. The total microwave electric field can be written as $\vec{E}(x, y, z, t)=\nabla \Phi(x, y) \sin (\omega t-k z)$ where $(x, y)$ denote transverse coordinates, $z$ the coordinate along the wires, $t$ is time, $k=\omega / c$ is the wave number (related to the angular field frequency $\omega$ by the vacuum dispersion relation), $c$ is the light velocity, and the scalar function $\Phi(x, y)$ determines the spatial distribution of the electrostatic potential between the two cylinders. The magnetic field is given by $\vec{B}(x, y, z, t)=\hat{z} \times \nabla \Phi(x, y) \sin (\omega t-k z) / c$. As shown in Ref.[16], the electric field is given by the explicit expression

$$
\vec{E}=\frac{E_{0}}{4} \frac{\left(v^{2}-u^{2}+1 / 4\right) \hat{x}-2 u v \hat{y}}{\left(v^{2}+u^{2}-1 / 4\right)^{2}+v^{2}}
$$

where $u=x / \Delta, v=y / \Delta$, and

$$
\Delta=\frac{1}{D} \sqrt{\left(D^{2}-R_{1}^{2}-R_{2}^{2}\right)^{2}-\left(2 R_{1} R_{2}\right)^{2}}
$$

is the distance between the filamentary charges of the logarithmic potential theory, $R_{1}$ and $R_{2}$ are the cylinder radii, and $D$ is the distance between cylinder centers. The geometry of the system can be seen in Fig. 1.

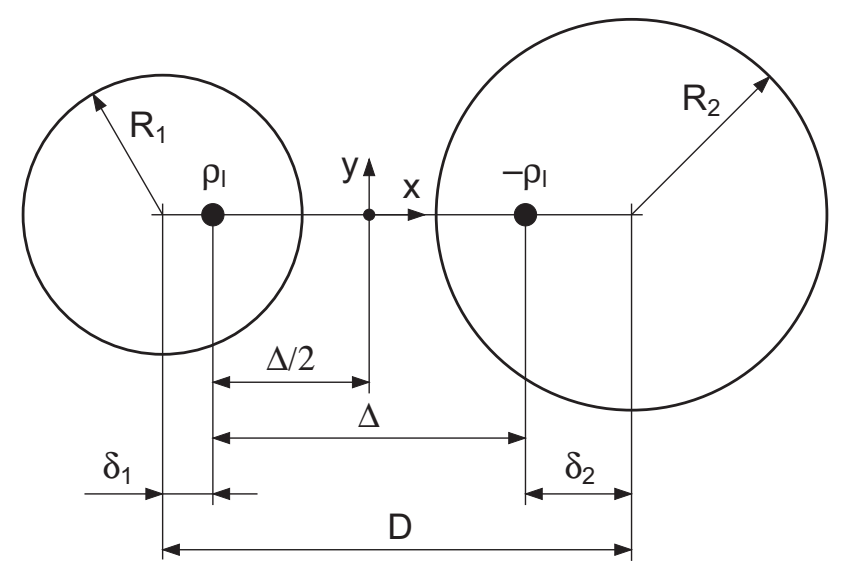

FIG. 1. The geometry of the two wire model, where $\rho_{l}$ and $-\rho_{l}$ denotes the filamentary line charge densities used in the logarithmic potential theory.

The quantity $E_{0}$ denotes the minimum electric field along the line connecting the cylinder centers and is given by

$$
E_{0}=\frac{4 V}{\Delta} \frac{1}{\operatorname{arcosh}\left(\frac{D^{2}-R_{1}^{2}-R_{2}^{2}}{2 R_{1} R_{2}}\right)}
$$


where $V$ is the voltage between the cylinders.

The formulas of the logarithmic potential theory are simplified considerably in the case of equal radii of the cylinders $\left(R_{1}=R_{2}=R\right)$, and the most relevant quantities can be summarized as follows:

$$
\begin{aligned}
& E_{0}=\frac{4 V}{\Delta} \frac{1}{\operatorname{arcosh}\left(\frac{D^{2}}{2 R^{2}}-1\right)} \\
& E_{\max }=\frac{E_{0}}{2}\left(1+\frac{D}{2 R}\right)
\end{aligned}
$$

where $E_{\max }$ denotes the maximum electric field between the cylinders, which is attained at the cylinder surfaces. Clearly the maximum electric field, $E_{\max }$, is related to the voltage, $V$, according to

$$
E_{\max }=\frac{V}{R} \sqrt{\frac{D+2 R}{D-2 R}} \frac{1}{\operatorname{arcosh}\left(\frac{D^{2}}{2 R^{2}}-1\right)}
$$

\section{OVERVIEW OF THE THEORY}

The theoretical model developed in Ref.[16] included two important effects; the geometrical dilution of electron density between gap passages due to the curved surfaces of the cylinders, and the spread of impact velocity caused by small deviations in the emission velocity coupled with the large number of field cycles required for an electron to cross the gap. Below we will briefly recapitulate these effects, and constrict them to the case of equal cylinder radii.

\section{A. Geometrical dispersion}

As discussed in Ref.[16], the two wire application involves an interesting and important loss mechanism caused simply by the geometry of the configuration in combination with the fact that the electric field is strongest at the cylinder surfaces and directed perpendicularly to these surfaces. This implies that secondary emitted electrons experience a strong acceleration close to the (curved) surfaces of emission and then drift radially outwards. Some of these electrons will impact the other cylinder surface where they in turn will cause emission of new secondary emitted electrons at impact. This process is then repeated on the return trip. Due to the geometrical spreading of the emitted electrons, caused by the curvature of 
the emitting surfaces and the radial acceleration, the electron density decreases after each passage between the cylinders, an effect that is equivalent to a loss of electrons. In a parallel plates geometry, there is no dispersion of electrons, and multipactor becomes possible when the secondary emission yield for impacting electrons is higher than unity, i.e.

$$
\sigma\left(v_{i}\right)>1
$$

where $v_{i}$ is the impact speed, and $\sigma$ is the secondary emission yield (SEY). The velocity where the SEY equals unity is typically called the first cross over velocity, $v_{1}$. In the two cylinder geometry this criterion becomes different. Since the electron density is diluted upon every passage, the net result can be characterized by an effective secondary emission coefficient, $\sigma_{\text {eff }}$, given by (assuming cylinders of the same material and equal radii, $R_{1}=R_{2}=R$ )

$$
\sigma_{\text {eff }}=\frac{\sigma\left(v_{i}\right)}{1+d / R}
$$

where $d$ is the distance between the cylinder surfaces $(d=D-2 R)$. The necessary condition for multipactor to occur becomes $\sigma_{\text {eff }}>1$, which implies $\sigma\left(v_{i}\right)>1+d / R$ i.e. a more restrictive condition than the classical multipactor condition $\sigma\left(v_{i}\right)>1$. The dispersive effect should be well pronounced in systems where the cylinder radii are small compared to the gap between the cylinder surfaces, $d / R \gg 1$, whereas in the large radii limit where $d / R \ll 1$ the parallel plates case, with minimal dispersion, is regained. The importance of the dispersive effect is easily assessed by noting that for the case of two cylinders with radii $\mathrm{R}=0.6 \mathrm{~mm}$ and a distance between the cylinder surfaces of $\mathrm{d}=3.8 \mathrm{~mm}$ (these dimensions are characteristic of an $8 \mathrm{GHz}$ helix antenna used for satellite communications), the requirement for multipactor breakdown becomes $\sigma\left(v_{i}\right)>1+d / R \approx 7.3$. This implies that multipactor breakdown in antennas with parameters of the same order as in the example should be unlikely, since most materials used in space applications have maximum values of the secondary emission coefficient of the order of 2 or 3.

\section{B. Resonant and non-resonant dynamics}

Electrons emitted from a surface will receive an initial acceleration due to the electric field. In a homogeneous electric field on the form

$$
E(t)=E_{m} \sin \omega t
$$


the electrons will move according to

$$
v(t)=v_{\omega} \cos \omega t+v_{e}-v_{\omega} \cos \omega t_{e}
$$

where $E_{m}$ is the field amplitude, $v(t)$ the electron velocity, $v_{\omega}=e E_{m} / m \omega$ is the oscillatory velocity amplitude, $e$ the electron charge, $m$ the electron mass, and we have used the initial condition $v\left(t_{e}\right)=v_{e}$, where $v_{e}$ is the electron emission velocity, and $t_{e}$ is the emission time.

This motion is explicitly separated into an oscillatory part (described by the first term on the RHS of Eq.(10)) and a drift one (given by the two last terms on the RHS of Eq.(10)). The drift velocity $v_{d}=v_{e}-v_{\omega} \cos \left(\omega t_{e}\right)$ is constant in this case and its value is determined by the emission velocity and the emission phase, $w t_{e}$, at fixed value of $v_{\omega}$. The impact electron velocity, $v_{i}=v_{\omega} \cos \left(\omega t_{i}\right)+v_{d}$, depends also on the impact phase, $\omega t_{i}$, and its maximum value, $v_{i, \max }=2 v_{\omega}+v_{e}$, is realized in the case of multipactor resonance when the emission phase, $\omega t_{e}=\pi$, and the electron flight time, $t_{i}-t_{e}$, equals an odd number of half RF cycles. A substitution of the maximum value of electron impact velocity into inequality (7) determines the lower threshold envelope for the multipactor resonance zones $^{20}$ which can be estimated using (7), with the approximation $v_{i} \approx 2 v_{\omega}$ in case of small emission velocity, $v_{e} \ll v_{\omega}$. However, even a small spread of emission velocity can result in a considerable spread of electron impact time when the gap width is large and the resonant flight time of electron considerably exceeds the rf period. This effect will blur the multipactor resonances and the inequality $(7)$ with the approximation $v_{i} \approx 2 v_{\omega}$ is non-appropriate to estimate the multipactor threshold. Instead, in a non-resonant discharge, it is more realistic to use an average value for the impact speed. As was discussed in Ref.[16], the probability for an electron to impact during a time interval, $P(d t)$, is equal to the distance traveled during that interval divided by the total distance covered by the electron in an entire period, that is

$$
P(d t)=\frac{v(t) d t}{\int_{0}^{T} v(t) d t}
$$

where $T$ is the RF period. This approach is valid also in case of a non uniform RF field taking into account the modification which is caused by variations both in amplitude of the electron oscillatory velocity, $v_{\omega}$, related to the local electric field amplitude, and the electron drift velocity governed by ponderomotive (Miller) force ${ }^{3,4,14}$. Specifically, as was shown in Ref.[16], equation (10) can be used (in case of $R_{1}=R_{2}$ ) to describe the temporal dependence 
of the electron velocity close to the impact point when the latter is not far from the straight line between the cylinder axis. It was also assumed in Ref.[16] that the main contribution to the multipactor discharge comes from the most energetic electrons $\left(\omega t_{e}=\pi\right)$, and that the emission velocity is small and can be neglected $\left(v_{e} \ll v_{\omega}\right)$, in equation (10). With these approximations (the corresponding applicability will be discussed in more details in Section $\mathrm{V})$, the impacting electrons have a velocity

$$
v_{i}(t)=v_{\omega} \cos \omega t+v_{\omega}
$$

where the probability of impact at a time $t$ depends on the velocity at that time. The probability for impact in an interval, $P(d t)=p(t) d t$, now becomes

$$
p(t) d t=\frac{\left(v_{\omega} \cos \omega t+v_{\omega}\right) d t}{\int_{0}^{T}\left(v_{\omega} \cos \omega t+v_{\omega}\right) d t}
$$

and the average impact velocity is

$$
\left\langle v_{i}\right\rangle=\int_{0}^{T} v(t) p(t) d t=\frac{\int_{0}^{T}\left(v_{\omega} \cos \omega t+v_{\omega}\right)^{2} d t}{\int_{0}^{T}\left(v_{\omega} \cos \omega t+v_{\omega}\right) d t}=\frac{3}{2} v_{\omega}
$$

In the language of classical multipactor theory, the necessary criterion for non-resonant multipactor is a high $f d$-product, where $f$ is the field frequency and $d$ the gap width between multipacting surfaces. More generally we can state that the discharge will be non-resonant when the spread in emission velocity is large enough to cause a spread in arrival time at the opposing surface equal to the field period, or equivalently when the distance traveled due to the emission velocity equals the electron oscillation amplitude. To express this criterion mathematically we note that in a resonant discharge with small emission velocity, the gap is traversed in an odd number, $N$, of RF cycles, i.e. $d \approx N \pi v_{\omega} / \omega$. The influence of the emission velocity on the resonance remains small as long as $v_{e} N T \ll v_{\omega} T$ or $v_{e} \ll v_{\omega}^{2} \pi / \omega d$. In the same way the discharge will be non-resonant when $v_{e} \gg v_{\omega}^{2} \pi / d \omega$ or $d \gg v_{\omega}^{2} \pi / v_{e} \omega$. For a more detailed discussion see Ref.[20]. 


\section{Application to the two cylinder system}

The discussion above implies that it is possible to use four different approximate models to estimate the breakdown threshold in the two cylinder system. When the gap width is small, and the cylinder radii is large, the situation is equivalent to that of resonant multipactor between two parallel plates, and we can use $v_{i} \approx 2 v_{\omega}$ and $\sigma_{\text {eff }} \approx \sigma\left(v_{i}\right)$, and determine the threshold at $\sigma\left(2 v_{\omega}\right)=1$. This approach is henceforth called the resonant non-dispersive approximation. Keeping the gap width small but decreasing the cylinder radii will increase the electron losses due to dispersion, and it is necessary to include this in the estimation of the effective SEY, i.e. $\sigma_{\text {eff }}=\sigma\left(v_{i}\right) /(1+d / R)$, but we keep the resonant approximation for the impact velocity, $v_{i} \approx 2 v_{\omega}$. This method will be called the resonant dispersive approximation. When both the gap width and the cylinder radii is large the discharge will be non-resonant and non-dispersive, and we should use $v_{i} \approx\left\langle v_{i}\right\rangle \approx 3 v_{\omega} / 2$ together with $\sigma_{\text {eff }} \approx \sigma\left(v_{i}\right)$. This will be called the non-resonant non-dispersive approximation. Finally, when the gap is wide, and the radii are small, we should use the non-resonant impact speed, $v_{i} \approx 3 v_{\omega} / 2$ along with the dispersive effective secondary emission coefficient $\sigma_{\text {eff }}=\sigma\left(v_{i}\right) /(1+d / R)$. This will be called the non-resonant dispersive approximation. The nomenclature, together with the proper criterias for when to use different combination of parameters, is summed up in Table 1.

\begin{tabular}{|c|c|c|}
\hline & $\sigma\left(v_{i}\right)=1,(R \gg d)$ & $\sigma\left(v_{i}\right)=1+d / R,(R$ arbitrary $)$ \\
\hline$v_{i} \approx 3 v_{\omega} / 2$ & Non-resonant, non-dispersive & Non-resonant, dispersive \\
$\left(d \gg \pi v_{\omega}^{2} / \omega v_{e}\right)$ & & \\
\hline$v_{i} \approx 2 v_{\omega}$ & Resonant, non-dispersive & Resonant, dispersive \\
$\left(v_{e} \ll \pi v_{\omega}^{2} / \omega d \approx v_{\omega} / N\right)$ & & \\
\hline
\end{tabular}

Table 1. The four different combinations of approximations for the multipactor threshold SEY and impact velocity of multipacting electrons, along with the criterias for when to use the different models in (parentheses).

Looking at Table 1 it is obvious that the non-dispersive approximation is just the limiting case of the dispersive aproximation when is $R$ very large. This can also be seen quite clearly in Figs. 2 and 4. 
To show the importance of the dispersive effect and the inhomogeneity of the electric field, a comparison between the predictions of the non-dispersive, and the dispersive approximation with a simplified field structure is shown in Fig.2. The breakdown voltages are calculated for silver, where $\sigma_{\max }=2.22$ (from Ref.[21]), using the Vaughan model for the $\mathrm{SEY}^{17}$, and the $\mathrm{y}$-axis shows the dispersive voltage, $V_{d}$, divided by the non-dispersive voltage, $V_{n d}$, as a function of normalized radii, $r=R / D$ (the ratio $V_{d} / V_{n d}$ is independant of the choice of impact velocity, thus both the non-resonant and resonant models are represented in Fig.2, see Ref. [16]). For $V_{d}$ the electric field used to calculate the oscillatory velocity is $E_{\max }$, the maximum field found on the two points on the cylinder surfaces which intersect the line connecting the cylinder centers, and the voltage between the cylinders is related to this field through Eq.(6). For the non-dispersive threshold, the voltage between the cylinders is divided by the gap width to estimate the electric field, $V / d=E$, and this estimate is used for calculating the oscillatory velocity. This way of estimating the threshold is quite close to the procedure recommended by the ESA standard for space applications ${ }^{21}$, which consists of relating the voltage to the electric field through the gap width, and then draw a Hatch-Williamson chart which shows all the combinations of voltage and frequency times gap width that are susceptible to multipactor breakdown. The lower envelope of such a chart will not be far from the estimate $V_{n d}$. It is clear that as the radii decreases, the breakdown voltage increases, to reach a maximum at $r_{\min }=1 /\left(\sigma_{\max }+1\right)=1 / 3.22 \approx 0.31$. Below this radius, double sided multipactor becomes impossible, according to this model.

The dilution of the electron density induced by the finite curvature of the emitting surface is similar to the effect of an angular velocity spread of the emission velocity ${ }^{5,19}$. This gives rise to a random walk of the electron trajectories between two parallel plates, and if the plates have finite extension, this effect ultimately makes the electrons go outside the plates and be lost. Furthermore, the angular spread of emission velocity also gives rise to an angular spread of impact velocity. In a more rigorous analysis of the two wire configuration, the effects of velocity spread should also be included and would clearly tend to further enhance the degradation of the secondary emission yield. In addition to this, the use of the average impact speed in the expression for the SEY is not quite correct. For the equality $\sigma\left(\left\langle v_{i}\right\rangle\right)=\left\langle\sigma\left(v_{i}\right)\right\rangle$ to hold, the dependance of the SEY-function on impact velocity should be linear, which is not generally true. A more detailed analysis should take this into account. Finally, another mechanism that has been neglected in the present analysis is the "transverse" component 


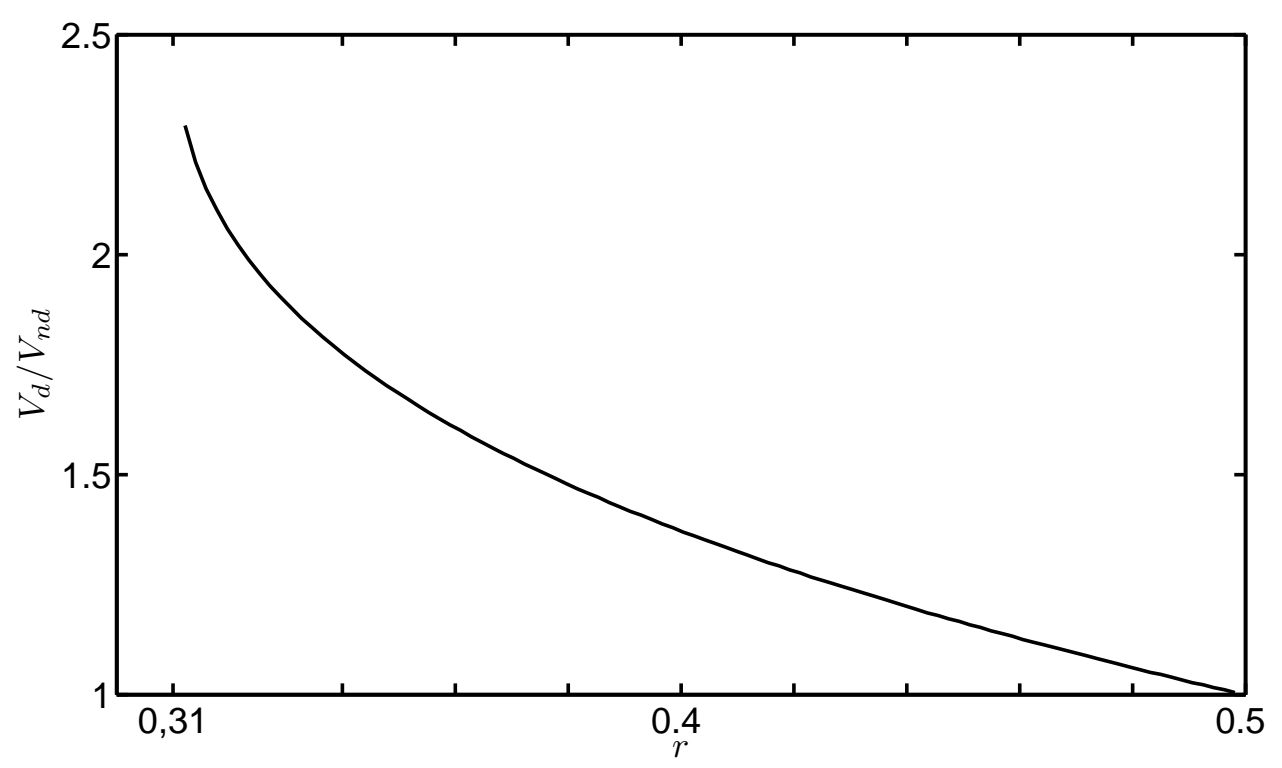

FIG. 2. The variation of the ratio $V_{d} / V_{n d}$ with normalized radius, $r=R / D$, using Vaughan's approximation for the secondary emission yield ${ }^{17}$, and using $\sigma_{\max }=2.22$, which is the ESA standard for silver ${ }^{21}$.

of the ponderomotive force that also should lead to an enhanced spread of the transverse width of the electron bunches during transits and to concomitant electron losses.

\section{FULL NUMERICAL SIMULATIONS}

An extensive numerical effort was done in order to simulate the multipactor avalanche in the complete two wire system. A software based on a Monte Carlo algorithm was developed. The exact form of the electric field was used, and the magnetic force on the electrons was neglected under the assumption that the electron velocity is small in comparison with the speed of light, and that the distance between cylinders is small compared to the RF wavelength. In addition to this simplification, only the two dimensional motion (in the plane which is perpendicular to the axis of the cylinders) of the electrons was considered. Space charge effects were not included, since only the first stage of the multipactor discharge is considered. A large number (up to $10^{5}$ ) of electron trajectories was calculated during a fixed interval of time, long enough to provide many gap crossings between the cylinders. The Euler method with a time step of $0.005 T$, where $T$ is the field period, was used for the 
integration of the electron trajectories. At the start of the simulation, all trajectories start at the same point - the intersection between the cylinder surface and the line connecting the cylinder centers, corresponding to the maximum field. The emission electron energy was fixed to $3 \mathrm{eV}$, with a direction of the velocity distributed randomly and evenly over $180^{\circ}$, above the cylinder surface. Upon impact with a cylinder surface, the number of electrons in each trajectory was multiplied with the secondary emission yield corresponding to the impact velocity given by Vaughan's model ${ }^{17}$

$$
\begin{aligned}
\sigma & =\sigma_{m}[\epsilon \exp (1-\epsilon)]^{\alpha} \\
\epsilon & =\frac{W_{i m p}}{W_{m}}
\end{aligned}
$$

where $\alpha=0.62$ for $\epsilon \leq 1$ and $\alpha=0.25$ for $\epsilon>1$ and $\sigma_{m}$ and $W_{m}$ are material dependent parameters that characterize the maximum value of the secondary emission coefficient and the impact energy at which the maximum secondary emission is attained respectively. After impact the trajectory starts from the point of impact with an emission energy corresponding to $3 \mathrm{eV}$ with a direction away from the surface randomly distributed in two dimensions. The voltage threshold for the existence of multipactor was determined by observing the number of electrons in a rectangular box around the cylinders and looking for decay or growth of the total electron population.

The aim of the simulations is to understand the influence of the wire dimensions on the multipactor breakdown condition and to compare the corresponding results with those of the previous approximate theory. The simplest approach would be to keep the wire radii (assumed equal) constant and then to vary the distance between the wires to see the effect on the multipactor threshold. However, this approach is not quite appropriate since the simple theory is based on a number of approximations. In particular, one of these (the one based on a significant spread in electron impact time and velocity) is violated when the distance between the cylinders becomes small enough and the multipactor is not suppressed by the spread in electron initial velocity. In order to have a more easily assessed comparison it was decided to keep the gap between the wires constant and instead vary the wire radii. Three simulation series were completed using two different distances; $d=3.8 \mathrm{~mm}$ (a realistic value) and $d=0.15 \mathrm{~mm}$ (a very narrow gap, where a resonant multipactor is probable). In two series the ESA standard for silver was used with $\sigma_{\max }=2.22$ and a first cross over energy $W_{1}=30 \mathrm{eV}^{21}$. Furthermore, in order to investigate the effect of the nonlinear dependence 
of $\sigma$ on the impact electron energy, i.e. the quality of the approximation $\left\langle\sigma\left(v_{i}\right)\right\rangle \approx \sigma\left(\left\langle v_{i}\right\rangle\right)$, one more simulation series was done using the unrealistically high value $\sigma_{\max }=10$, but keeping the same value of the first cross over energy $\left(W_{1}=30 \mathrm{eV}\right)$. This has the advantage of making the secondary emission curve almost linear around the first cross over energy.

A first illustration of the calculated electron trajectories, demonstrating the dispersion of the trajectories and the resulting electron losses for different radii, is shown in Fig.3. It is clear that the dispersion of the trajectories actually becomes stronger than predicted by the geometric spreading mechanism of the simple theory when the cylinder radii decreases. This is evident from the bent electron trajectories seen in the upper figure. The acceleration outwards is due to the ponderomotive force working in the perpendicular direction with respect to the line between cylinder centers. This effect is not included in the simple theory.
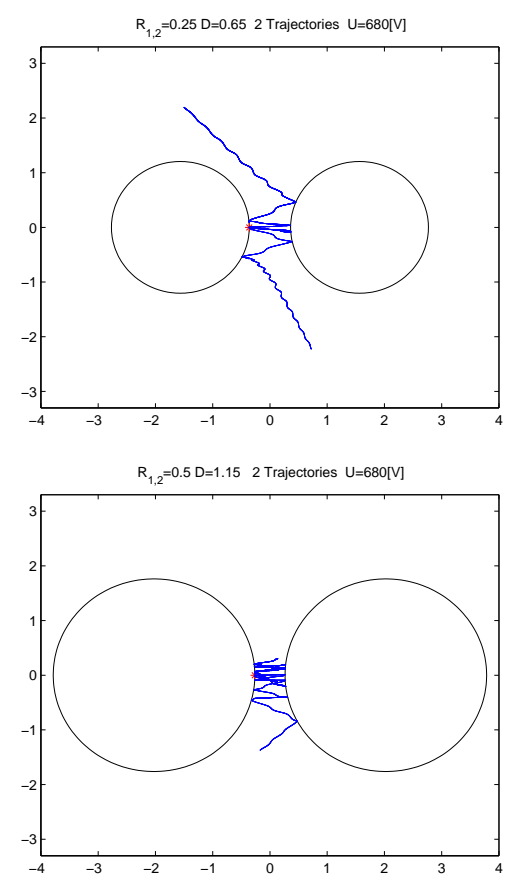

FIG. 3. Figure illustrating cases of weak, but noticeable dispersion (lower figure: $R=5 \mathrm{~mm}$ ) and strong dispersion (upper figure: $R=2.5 \mathrm{~mm}$ ) of electron trajectories. Distance between wires $d=1.5 \mathrm{~mm}$. Applied voltage $V=680$ volts.

The multipactor simulations were made for the frequency $f=8 \mathrm{GHz}$ and for two significantly different gap widths: $d=0.15 \mathrm{~mm}$, a small width that corresponds approximately to the first resonance zone and the larger (and more realistic) width $d=3.8 \mathrm{~mm}$ that corresponds to non-resonant multipactor. The results for the observed multipactor voltages are 
summarized in Tables 2, 3 and 4 .

\begin{tabular}{|c|c|}
\hline Wire radii $\left(R_{1}=R_{2}\right) \mathrm{mm}$ & $V$ Volt \\
\hline 30 & 70 \\
10 & 70 \\
3 & 70 \\
1 & 80 \\
0.5 & 90 \\
0.3 & 100 \\
\hline
\end{tabular}

Table 2. The multipactor threshold (in terms of the RF voltage amplitude, $V$ ) in a two wire system with small gap width $d=0.15 \mathrm{~mm}$, which corresponds approximately to the first resonance zone, and $\sigma_{\max }=2.22$. In this case multipactor breakdown was found to be impossible for wire radii less than $0.3 \mathrm{~mm}$. On the other hand, the multipactor breakdown voltage was found to be independent of the wire radii when the latter exceeded about $3 \mathrm{~mm}$.

\begin{tabular}{|c|c|}
\hline Wire radii $\left(R_{1}=R_{2}\right) \mathrm{mm}$ & $V$ Volt \\
\hline 30 & 3800 \\
20 & 3800 \\
15 & 4000 \\
10 & 5800 \\
8.5 & 7900 \\
\hline
\end{tabular}

Table 3. The multipactor threshold (in terms of the RF voltage amplitude, $V$ ) in a two wire system with gap width $d=3.8 \mathrm{~mm}$, a more realistic width for an $8 \mathrm{GHz}$ antenna, and $\sigma_{\max }=2.22$. In this case multipactor breakdown was found to be impossible for wire radii less than $8.5 \mathrm{~mm}$. On the other hand, the multipactor breakdown voltage was found to be independent of the wire radii when the latter exceeded about $20 \mathrm{~mm}$. 


\begin{tabular}{|c|c|}
\hline Wire radii $\left(R_{1}=R_{2}\right) \mathrm{mm}$ & $V$ Volt \\
\hline 30 & 2800 \\
20 & 3100 \\
15 & 3300 \\
10 & 3800 \\
7.5 & 4400 \\
5 & 5400 \\
\hline
\end{tabular}

Table 4. The multipactor threshold (in terms of the RF voltage amplitude, $V$ ) in a two wire system with gap width $d=3.8 \mathrm{~mm}$ and $\sigma_{\max }=10$. In this case multipactor breakdown was found to be impossible for wire radii less than $5 \mathrm{~mm}$. On the other hand, the multipactor breakdown voltage was found to be independent of the wire radii when the latter exceeded about $30 \mathrm{~mm}$.

\section{COMPARISON BETWEEN SIMULATIONS AND THEORY}

The simulated results make it possible to assess the accuracy of the theoretical framework developed in Ref.[13], as well as that of the classical resonance theory. For a small gap width, we expect resonance theory to give a good estimate, whereas for wider gaps, the non-resonant approach should give a better agreement with the simulated data.

\section{A. Small gap}

The first simulation used a small gap width of $0.15 \mathrm{~mm}$. Given the value for the frequency, width, emission velocity and first cross over velocity already mentioned, we expect a multipactor discharge belonging to the first resonance zone. This is indeed confirmed in Fig.4, which presents the breakdown threshold predictions according to all four approximate methods presented in Table 1, as well as the simulated data. The simulations spanned the range of radii $R \in[0.3,30] \mathrm{mm}$. The smallest radius allowing multipactor according to the dispersive approximation is given by $\sigma_{\max } /\left(1+d / R_{\min }\right)=1$ or $R_{\min }=d /\left(\sigma_{\max }-1\right) \approx 0.12$ $\mathrm{mm}$.

The figure shows that the resonant approximation gives a good estimate for the voltage 


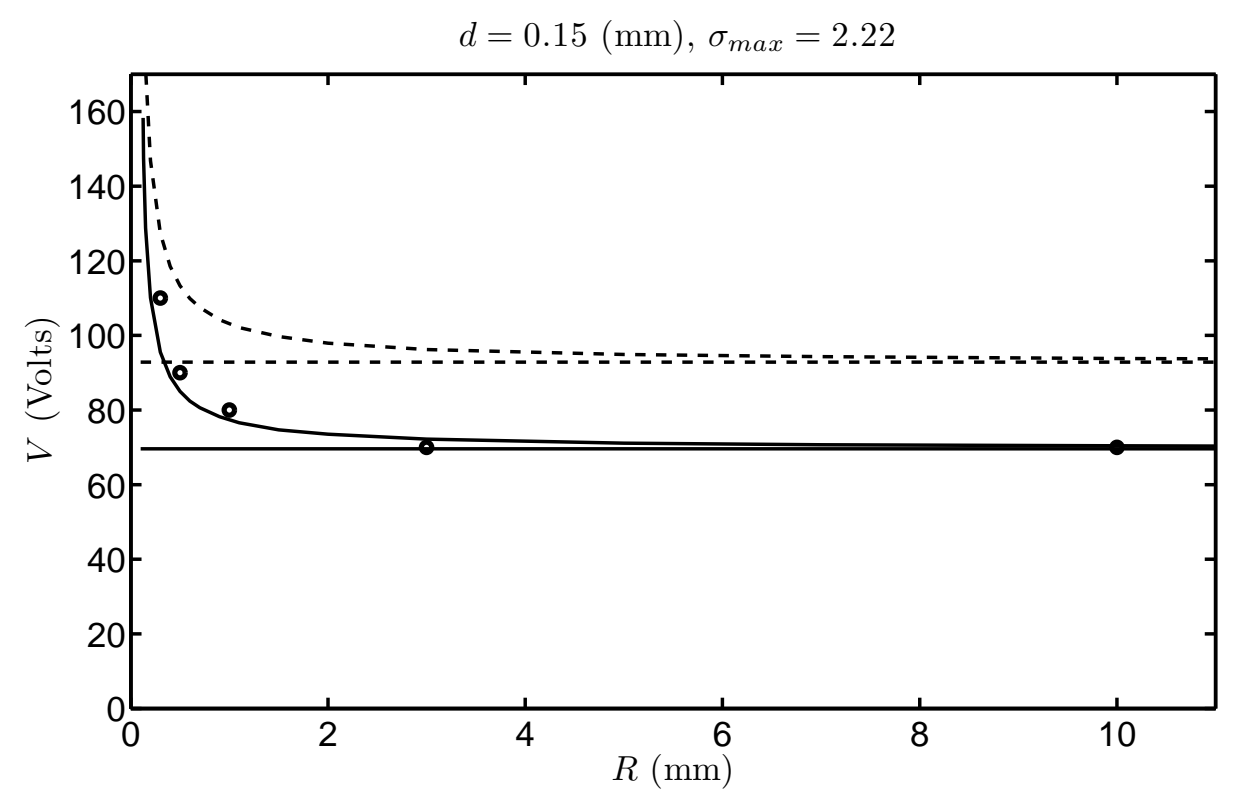

FIG. 4. Multipactor breakdown thresholds for $d=0.15 \mathrm{~mm}$ and $\sigma_{\max }=2.22$. The straight solid line corresponds to the lower breakdown threshold according to the resonant non-dispersive approximation, whereas the dashed straight line corresponds to non-resonant non-dispersive approximation. The circles represent the simulated data. The curved solid line corresponds to the predictions of the resonant dispersive approximation, and the dashed curved line to those of the non-resonant dispersive approximation

threshold, and that as the radii decreases, the dispersive losses become more and more important, as can be seen by the widening gap between the curved and straight solid lines. The non-resonant approximation produces a too high threshold, simply due to the resonant behavior of the electrons, where impact will occur only during the most energetic phase and electrons will tend to bunch up around this phase due to phase focusing mechanisms. In the non-resonant approximation the electrons are assumed to impact during the entire field period and to yield an average impact velocity of $3 v_{\omega} / 2$, which in this case is lower than the actual impact velocity. The most interesting part of the curve is the left side. Both the simulated data and the prediction of the dispersive approximation start to rise rapidly with decreasing radius. This indicates that there is a geometrical limit radius below which double sided multipactor becomes impossible, a feature that the non-dispersive approximation does not include, and which at the same time is a very important result for RF system design considerations. 


\section{B. Large gap}

The second simulation used a large gap width of $d=3.8 \mathrm{~mm}$, the same as for the 8 $\mathrm{GHz}$ antenna mentioned above. The multipacting electrons will perform many oscillations while traversing the gap, and even a small spread in electron emission phase will cause the electron impact phase to be randomized. Thus, resonant behavior will be strongly suppressed. The non-resonant approximation assumes a random impact phase, whereas the resonant approximation assumes electrons to impact only during the most energetic phase. We should therefore expect the resonant approximation to produce a threshold which is too low, whereas the non-resonant approximation should give a better agreement with simulations. Fig.5 summarizes all relevant data relating to this simulation.

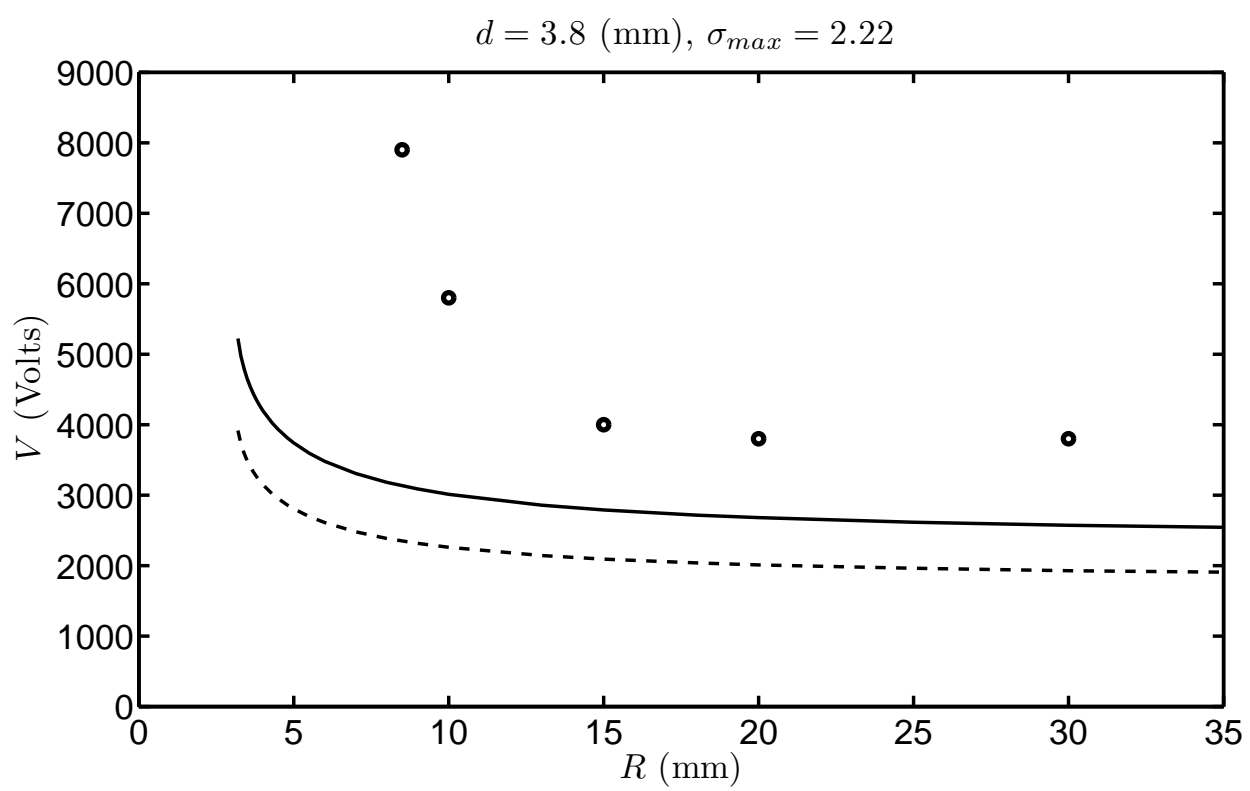

FIG. 5. Multipactor breakdown threshold for $d=3.8 \mathrm{~mm}$ and $\sigma_{\max }=2.22$. The circles represent the simulated data. The solid curved line corresponds to the predictions of the non-resonant dispersive approximation, and the dashed curved line to those of the resonant dispersive approximation.

It is clear that both the resonant and non-resonant approximations tend to predict a too low breakdown threshold, although the non-resonant less so. In the limit of large radii (right side of the figure), the losses due to the curvature of the cylinder surfaces should be small and the reason for the discrepancy between the non-resonant dispersive approximation and simulations must be found elsewhere. In the process of estimating the impact velocity it was assumed that the emission velocity was small and could be neglected. However, the 
simulations use a rather high value of emission velocity, and in a more accurate analysis this velocity should be included. It is quite clear though that introducing an extra velocity component into the approximate formulas for the impact velocity will only serve to lower the necessary oscillatory velocity for multipactor avalanche to occur. This in turn decreases the threhold voltage, and increases the discrepancy between the approximate models and the simulations. Another source of errors lies in the fact that the non-resonant approximation treats the dependance of the secondary emission yield on the impact velocity as linear.

To investigate the effect of the nonlinearity of the secondary emission curve, a third simulation used an unrealistically high secondary emission yield, $\sigma_{\max }=10$, while keeping the same first cross over velocity as before, $W_{1}=30 \mathrm{eV}$. This makes the secondary emission curve more linear around the first cross over point, and should decrease the error introduced by the direct use of the average impact velocity. Since the position of the first cross over point was not changed, the predicted voltage threshold in the large radii limit remains the same as that for $\sigma_{\max }=2.22$, for both the non-resonant and resonant approximation, therefore only the non-resonant curve is shown in Fig.6. The minimum radii where the curve terminate to the left is shifted to a lower value as compared to Fig.5.

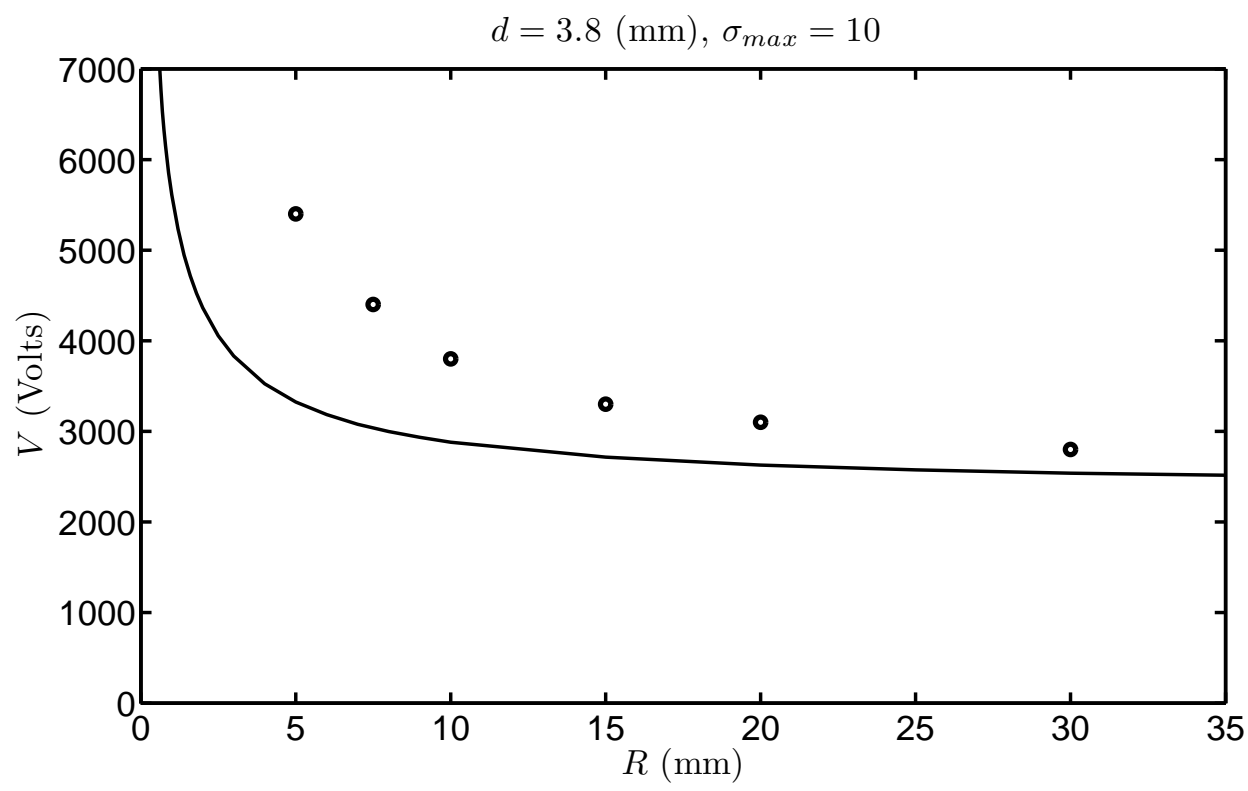

FIG. 6. Multipactor breakdown threshold for $d=3.8 \mathrm{~mm}$ and $\sigma_{\max }=10$. The circles represent the simulated data. The curved line corresponds to the predictions of the non-resonant dispersive approximation.

Fig.6, implies that increasing the secondary emission yield to very high values does lower 
the simulated multipactor breakdown threshold and makes it come closer to the predictions by the non-resonant dispersive approximation (as compared to those for $\sigma_{\max }=2.22$ ). This could be due to the suppression of the nonlinearity of the secondary emission function. However, there is still a notable difference between the simple theory and the simulations. This is most likely due to the combined effect of ponderomotive spreading in the tangential direction, the spread in electron emission angle, and the fact that not only the most energetic electrons will be involved in the multipactor discharge, but there will rather be a range of electron energies impacting the surface, thus lowering the average impact energy further.

\section{CONCLUSION}

In a previous analysis of ours ${ }^{16}$, we considered multipactor breakdown between two parallel infinite cylinders, being a very simplified, but still relevant, model of a helix antenna. This model geometry has the advantage that the electric field configuration is available in closed analytical form, which simplifies analytical as well as numerical analysis of the multipactor dynamics. The electron trajectories in this field were analyzed using a simple, but useful, model where the electron motion is divided into a slowly varying drift velocity (driven by the ponderomotive force due to the electric field inhomogeneity) and a rapidly oscillating part (driven by the oscillating electric field). This made it possible to draw several important conclusions: electrons emitted at one cylinder surface are strongly accelerated in the field closest to the surface and then drift towards the other cylinder surface. During this drift, the electrons tend to spread out due to the curvature of the surface of emission, a process that is again repeated on the way back to the original surface after the electrons have struck the other cylinder and been re-emitted. This phenomenon is equivalent to a loss of electrons and leads to a more stringent multipactor condition than that of the classical case of two plane parallel infinite plates. The importance of this effect is determined by the ratio between the distance between the cylinders and the cylinder radii, and a preliminary estimate showed that when this ratio is large, multipactor can only occur for surfaces having very large secondary emission coefficients. A more detailed analysis was also made in order to assess the lowest voltage between the cylinders at which multipactor becomes possible (although it may not necessarily occur) i.e. the critical voltage between the wires below which multipactor breakdown is impossible. This critical voltage was found to be a function 
of all geometrical dimensions of the system as well as of the frequency of the electric field.

In the present work, full simulations of the multipactor breakdown problem have been made in order to evaluate the accuracy of the previously presented simple model. It is found that the predictions of the model are in good qualitative agreement with the results of the simulations. In the limit of small gap between cylinder surfaces and large cylinder radii, the non-resonant approximation overestimates the breakdown threshold, because of the averaging over impact phases of the electrons, when really the resonant approximation should be used. However, when the radii decrease, the simulated threshold, as well as the theoretically predicted threshold according to the dispersive approximation, starts to increase, and eventually terminates at a certain radius determined by the maximum secondary emission yield, indicating that no double sided multipactor breakdown is possible, regardless of the voltage. This feature is not predicted by the classical non-dispersive approximation and is an important fact to consider when designing this type of transmission systems. In the other limit, when the gap width is quite large, the non-resonant approximation tends to underestimate the breakdown threshold. However, the error is much smaller than that corresponding to resonant approximation, and the qualitative agreement in the limits of both small and large gap widths indicates that the model incorporates the most important electron loss mechanisms.

1 A. M. Perez, C. Tienda, C. Vicente, A. Coves, G. Torregrosa, B. Gimeno, R. Barcot, V. E. Boria and D. Raboso, "Multipactor analysis in coaxial waveguides for satellite applications using frequency-domain methods", 2006 IEEE MTT-S International Microwave Symposium Digest, 1045, (2006)

2 M. N. Buyanova, V. E. Nechaev, and V. E. Semenov, "Multipactor discharge on a dielectric surface in the field of circularly polarized plane waves", Radiophysics and Quantum Electronics, $50,893(2007)$

3 V. E. Semenov, E. I. Rakova, D. Anderson, M. Lisak and J. Puech, "Multipactor in rectangular waveguides", Phys. Plasmas 14, 033501 (2007)

4 R. Udiljak, D. Anderson, M. Lisak, V. E. Semenov and J. Puech, "Multipactor in a coaxial transmission line, part 1: analytical analysis", Phys. Plasmas 14, 033508 (2007) 
5 A. G. Sazontov, V. A. Sazontov, and N. K. Vdovicheva, "Multipactor breakdown prediction in a rectangular waveguide: statistical theory and simulation results", Contrib. Plasma Phys. 48, $331(2008)$

${ }^{6}$ F. Pérez, J. de Lara, L. Conde, M. Alfonseca, L. Galán, D. Raboso, "CEST and MEST: Tools for the simulation of radio frequency electric discharges in waveguides" in Simulation Modelling Practice and Theory (Elsevier), Vol 16(9), 1438 (2008)

7 V. E. Semenov, E. Rakova, R. Udiljak, D. Anderson, M. Lisak and J. Puech, "Conformal mapping analysis of multipactor breakdown in waveguide irises", Phys. Plasmas 15, 033501 (2008)

8 O. V. Sinitsyn, G. S. Nusinovich, and T. M. Antonsen, Jr., "Self-consistent nonstationary twodimensional model of multipactor in dielectric-loaded accelerator structures", Phys. Plasmas, 16, 073102, (2009)

9 A. M. Pérez, V. E. Boria, B. Gimeno, S. Anza, C. Vicente, J. Gil, "Multipactor analysis in circular waveguides", J. Electromagnetic Waves and Applications, 23, 1575 (2009)

10 V. E. Semenov, E. I. Rakova, A. G. Sazontov, I. M. Nefedov, V. I. Pozdnyakova, I. A. Shereshevskii, D. Anderson, M. Lisak, and J. Puech, "Simulations of multipactor thresholds in shielded microstrip lines", J. Phys. D: Appl. Phys. 42, 205204 (2009)

11 V. E. Semenov, N. A. Zharova, D. Anderson, M. Lisak and J. Puech, "Simulations of multipactor in circular waveguides", Phys. Plasmas 17, 123503 (2010)

12 V. E. Semenov, N. Zharova, R. Udiljak, D. Anderson, M. Lisak, and J. Puech, "Multipactor in a coaxial transmission line. II. Particle-in-cell simulations", Phys. Plasmas, 14, 033509 (2007)

13 A. M. Perez, C. Tienda, C. Vicente, S. Anza, J. Gil, B. Gimeno, V. E. Boria, D. Raboso, "Prediction of multipactor breakdown thresholds in coaxial transmission lines for traveling, standing, and mixed waves", IEEE Trans. Plasma Science, 37, 2031 (2009)

14 V. E. Semenov, E. Rakova, N. Zharova, D. Anderson, M. Lisak, and J. Puech, "Simulations of the multipactor effect in hollow waveguides with wedge-shaped cross section", IEEE Trans. Plasma Science, 36, 488 (2008)

15 J. Hueso, C. Vicente, B. Gimeno, V. E. Boria, S. Marini, M. Taroncher, "Multipactor effect analysis and design rules for wedge-shaped hollow waveguides", IEEE Trans. Electron Devices, $57,3508(2010)$

16 J. Rasch, D. Anderson, J. F. Johansson, M. Lisak, J. Puech, E. Rakova and V. E. Semenov, 
"Microwave multipactor breakdown between two cylinders", IEEE Trans. Plasma Science, 38, $1997(2010)$

17 J. R. M. Vaughan, " A new formula for secondary emission yield", IEEE Trans. Electron Devices, $36,1963(1989)$

18 B. D. Popovich, "Introductory engineering electromagnetics", Addison-Wesley, Reading Massachusetts, USA (1971)

19 A. Sazontov, M. Buyanova, V. Semenov, E. Rakova, V. Vdovicheva, D. Anderson, M. Lisak, J. Puech, and L. Lapierre, "Effect of emission velocity spread of secondary electrons in two-sided multipactor", Phys. Plasmas, 12, 053102 (2005)

20 A. Kryazhev, M. Buyanova, V. E. Semenov, D. Anderson, M. Lisak, J. Puech, L. Lapierre, and J. Sombrin, "Hybrid resonant modes of two-sided multipactor and transition to the polyphase regime", Phys. Plasmas, 9, 4736 (2002)

21 ESA for ECSS, "Space engineering, multipactor design, and test." ESA Publications Division, Noordwijk, The Netherlands, ECSS-E-20-01-01A, 5 May (2003) 